\title{
Azithromycin-resistant Neisseria gonorrhoeae isolates in Guangzhou, China (2009-2013): coevolution with decreased susceptibilities to ceftriaxone and genetic characteristics
}

Jing-Yao Liang ${ }^{1,2+}$, Wen-Ling Cao ${ }^{1,2+}$, Xiao-Dong $\mathrm{Li}^{1,2}$, Chao Bi ${ }^{1,2}$, Ri-Dong Yang ${ }^{1,2}$, Yan-Hua Liang ${ }^{1,2}$, Ping Li ${ }^{1,2}$, Xing-Dong Ye ${ }^{1,2}$, Xiao-Xiao Chen ${ }^{1,2}$ and Xi-Bao Zhang ${ }^{1,2^{*}}$

\begin{abstract}
Background: The recent emergence of azithromycin-resistant (AZM-R) N. gonorrhoeae isolates that have coevolved decreased susceptibility to extended-spectrum cephalosporins has caused great concern. Here we investigated the prevalence of decreased susceptibility to ceftriaxone $\left(C R O^{D}\right)$ in $A Z M-R$ isolates and genetically characterized AZM-R isolates in Guangzhou, China from 2009 to 2013.

Methods: The minimum inhibitory concentration (MIC) of AZM and ceftriaxone was determined using an agar-dilution method. All AZM-R isolates were screened for mutations in $23 \mathrm{~S}$ rRNA, $m$ trR and penA genes and genotyped using $\mathrm{N}$. gonorrhoeae multi-antigen sequence typing (NG-MAST).

Results: Of the 485 identified N. gonorrhoeae isolates, 445 (91.8\%) were isolated from male urethritis subjects, and 77 (15.9\%) were AZM-R (MIC $\geq 1 \mathrm{mg} / \mathrm{L})$, including 33 (6.8 \%) with AZM low-level resistant (AZM-LLR, MIC = $1 \mathrm{mg} / \mathrm{L}$ ) and 44 (9.1\%) with AZM middle-level resistant (AZM-MLR, MIC $\geq 2 \mathrm{mg} / \mathrm{L}$ ). Significantly more CRO ${ }^{\mathrm{D}}$ (MIC $\geq 0.125 \mathrm{mg} / \mathrm{L}$ ) showed in AZM-MLR isolates $(43.2 \%, 19 / 44)$ as compared with that in AZM-LLR isolates $(18.2 \%, 6 / 33)(p<0.05)$. For the $23 \mathrm{~S}$ rRNA, mtrR, penA or combined 23S rRNA/MtrR/penA mutations, no significant difference was found between AZM-LLR isolates and AZM-MLR isolates $(P>0.05)$; similar results were detected between combined AZM-LLR/CRO ${ }^{D}$ isolates and combined AZM-MLR/CROD isolates $(P>0.05)$. No mutation A2059G or AZM high-level resistant (AZM-HLR, MIC $\geq 256 \mathrm{mg} / \mathrm{L}$ ) isolate was detected. Among 77 AZM-R isolates, 67 sequence types (STs) were identified by NG-MAST, of which 30 were novel. Most STs were represented by a single isolate.
\end{abstract}

Conclusions: The AZM-R together $C R O^{\mathrm{D}}$ isolates are now present in Guangzhou, China, which deserve continuous surveillance and the mechanism of concurrent resistance needs further study.

Keywords: Neisseria gonorrhoeae, Azithromycin, Antimicrobial resistance, NG-MAST

\footnotetext{
*Correspondence: gzpfbfzs@yahoo.com

'Equal contributors

${ }^{1}$ Institute of Dermatology, Guangzhou Medical University, Guangzhou

510095, PR China

${ }^{2}$ Department of Dermatology, Guangzhou Institute of Dermatology, 56

Hengfu Road, Guangzhou 510095, PR China
} 


\section{Background}

The global spread of multidrug-resistant Neisseria gonorrhoeae ( $N$. gonorrhoeae) is a growing public health threat. Worryingly, clinical treatment failures with the extended-spectrum cephalosporins (ESCs) [1], the last remaining options for empirical first-line monotherapy, have recently been reported. Accordingly, dual antimicrobial therapies have been introduced in several countries [2, 3], including China [4]. These consist of ceftriaxone (250-500 mg intramuscularly) or cefixime (400 mg orally, if ceftriaxone is not an option) together with azithromycin (AZM, 1-2 g orally) for treatment of uncomplicated gonorrhea. AZM was recommended for patients with gonorrhea or coinfection with Chlamydia trachomatis in China around the year 2000 [5], and since then has been broadly used because of its wide availability and ease of administration. In China, AZM-resistant (AZM-R) N. gonorrhoeae were first identified during 2001-2003 [6], and the first AZM-R isolates were identified in Guangzhou in 2009 [7]. Treatment failures with $2 \mathrm{~g}$ of AZM and high-level AZM resistant $N$. gonorrhoeae isolates with a minimum inhibitory concentration $(\mathrm{MIC}) \geq 256 \mathrm{mg} / \mathrm{L}$ have been verified in several countries $[8,9]$. Recently, a surveillance of antimicrobial resistance in gonococci from Canada resulted in two high-level AZM-R isolates (MIC $\geq 2048 \mathrm{mg} / \mathrm{L}$ ), which may be due to mutation A2143G (N. gonorrhoeae numbering) in the four copies of the 23S rRNA gene [9].

Mutations at various genes such as 23S rRNA, mtrR and penA (encoding penicillin-binding protein $2, \mathrm{~PB}$ $\mathrm{P} 2)$, have been identified associating with chromosomally mediated resistance to AZM [10, 11] and ESCs [12] in $N$. gonorrhoeae. Globally, AZM-R together decreased susceptibility to ESCs in $N$. gonorrhoeae has only rarely been reported $[9,13]$. In this study, we investigated the prevalence of decreased susceptibility to ceftriaxone $\left(\mathrm{CRO}^{\mathrm{D}}\right)$ in AZM-R N. gonorrhoeae isolates, and the molecular characteristics of AZM-R N. gonorrhoeae isolates from January 2009 to November 2013 in Guangzhou, China.

\section{Methods}

\section{Gonococcal isolates and susceptibility testing}

Clinical $N$. gonorrhoeae isolates were consecutively collected from urethral or cervical specimen of one per patient with gonorrhea attending sexually transmitted infection (STI) clinics in Guangzhou, China between January 2009 and November 2013. The demographic and clinical information of patients such as gender, age, and symptoms was collected by completing a questionnaire. Each isolate was cultured, verified, and preserved as described [14]. MIC for AZM and ceftriaxone were determined by the agar dilution method, according to recommendations from the WHO [15]. Antimicrobial susceptibility for AZM was interpreted using the European Committee on Antimicrobial Susceptibility Testing (EUCAST) guidelines (http://www.eucast.org/), and ceftriaxone susceptibility was interpreted according to criteria defined by WHO in 2012 [15]. Briefly, isolates with $\mathrm{MIC} \geq 1.0 \mathrm{mg} / \mathrm{L}$ for $\mathrm{AZM}$ were classified as resistant, and isolates with $\mathrm{MIC} \geq 0.125 \mathrm{mg} / \mathrm{L}$ for ceftriaxone were classified as having decreased susceptibility. N. gonorrhoeae ATCC 49226 and strains G, L, and P of the 2008 WHO N. gonorrhoeae reference strain panel were used as quality control strains. To compare with a previous report [5] and better analyze the results, AZM-R $N$. gonorrhoeae isolates $(\mathrm{MIC} \geq 1.0 \mathrm{mg} / \mathrm{L})$ were divided into AZM low-level resistant (AZM-LLR) N. gonorrhoeae isolates $(\mathrm{MIC}=1.0 \mathrm{mg} / \mathrm{L}), \mathrm{AZM}$ middle-level resistant (AZM-MLR) N. gonorrhoeae isolates (MIC $\geq$ $2.0 \mathrm{mg} / \mathrm{L}$ ), and AZM high-level resistant (AZM-HLR) $N$. gonorrhoeae isolates (MIC $\geq 256 \mathrm{mg} / \mathrm{L})$ in this study based on the previous studies $[9,10]$.

\section{Sequence-based molecular epidemiologic studies}

Bacterial genome DNA from each identified AZM-R isolate was extracted using the TIANamp Bacterial DNA kit (TIANGEN, Beijing, China). As previously described, mutations in the four alleles of the 23S rRNA [16], mtrR and penA genes [12], and polymorphisms in porB and $t b p B$ [17] were amplified by PCR. The PCR products were purified and sequenced by Shenggong Co., Ltd. (Shanghai, China). DNA sequences were aligned using BLAST and GenBank programs (http://www.ncbi.nlm.nih.gov) to identify mutations in the 23S rRNA gene. DNA sequences of $m t r R$ and penA genes were translated into deduced amino acid sequences, which were then aligned with their respective prototypes in $N$. gonorrhoeae using Proteomics and Sequence Tools (http:// ca.expasy.org/). N. gonorrhoeae isolates were typed using the $N$. gonorrhoeae multi-antigen sequence typing (NGMAST) method [17]. NG-MAST allele numbers of por $B$ and $t b p B$ and STs were obtained using a publicly accessible database on the NG-MAST website (www.ng-mast.net). A phylogenetic tree was created using ClustalX software (version 1.83; http://www.clustal.org/download/ 1.X/ftp-igbmc.u-strasbg.fr/pub/ClustalX. html) and Mega software (version 6.0.5; http://www.megasoftware.net/ mega.php) based on the more variable segments of por $B$ (490 bp) and $t b p B(390 \mathrm{bp})$ as analyzed by NG-MAST.

\section{Statistical analysis}

Statistical significance was assessed using SPSS 13.0 (SPSS Inc, Chicago, IL). Chi-square tests were used for statistical analyses. A $P$-value $<0.05$ was considered to be significant. 
Table 1 The resistance rates for AZM in N. gonorrhoeae isolated in Guangzhou, China between 2009 and 2013

\begin{tabular}{|c|c|c|c|}
\hline \multirow[b]{2}{*}{ Year } & \multicolumn{2}{|l|}{$\mathrm{MIC}$} & \multirow[b]{2}{*}{ Total } \\
\hline & $1 \mathrm{mg} / \mathrm{L}$ & $\geq 2 \mathrm{mg} / \mathrm{L}$ & \\
\hline 2009 & $19.3 \%(17 / 88)$ & $4.5 \%(4 / 88)$ & $23.9 \%(21 / 88)$ \\
\hline 2010 & $8.4 \%(7 / 83)$ & $4.8 \%(4 / 83)$ & $13.3 \%(11 / 83)$ \\
\hline 2011 & $0.9 \%(1 / 114)$ & $7.9 \%(9 / 114)$ & $8.8 \%(10 / 114)$ \\
\hline 2012 & $2.0 \%(2 / 100)$ & $17.0 \%(17 / 100)$ & $19.0 \%(19 / 100)$ \\
\hline 2013 & $6.0 \%(6 / 100)$ & $10.0 \%(10 / 100)$ & $16.0 \%(16 / 100)$ \\
\hline Total & $6.8 \%(33 / 485)$ & $9.1 \%(44 / 485)$ & $15.9 \%(77 / 485)$ \\
\hline
\end{tabular}

\section{Ethics approval}

The study protocol was approved by the Medical Ethics Committee at the Institute of Dermatology, the Chinese Academy of Medical Sciences \& Peking Union Medical College and the National Center for Sexually Transmitted Disease Control, Nanjing, China (approval number 2011-KY-003). Written informed consent was obtained from all participating subjects.

\section{Results}

\section{Antimicrobial susceptibility testing}

A total of $485 \mathrm{~N}$. gonorrhoeae isolates were identified in Guangzhou from 2009 to 2013 (Table 1). Of these, the mean age of the patients was 31.6 years (range from 17 to 69 years), $91.8 \%(445 / 485)$ isolates were isolated from male patients with urethritis. Among all the isolates, $15.9 \%$ (77/485) showed AZM-R, including $6.8 \%$ (33/485) AZM-LLR and $9.1 \%(44 / 485)$ AZM-MLR. The proportion of AZM-LLR isolates decreased from $14.0 \%(24 / 171)$ in $2009-2010$ to $2.9 \%(9 / 314)$ in 2011-2013 $(p<0.05)$, whereas AZM-MLR isolates increased from $4.7 \%(8 / 171)$ in $2009-2010$ to $11.5 \%$ (36/ $314)$ in 2011-2013 $(p<0.05)$. In the 2011-2013 study period, seven isolates with MIC $>8 \mathrm{mg} / \mathrm{L}$ appeared for the first time and accounted for $2.2 \%$ (7/314) of the examined isolates (Fig. 1). The percentage of $\mathrm{CRO}^{\mathrm{D}}$ isolates was $22.3 \%(108 / 485)$ in all identified $N$. gonorrhoeae isolates, $18.2 \%(6 / 33)$ in AZM-LLR isolates, and $43.2 \%(19 / 44)$ in AZM-MLR isolates which was a significant increase compared with the percentage of all identified $N$. gonorrhoeae isolates or AZMLLR isolates (both $p<0.05$, Table 2).

\section{5 rRNA, mtrR and penA mutations in AZM-R N. gonor- rhoeae isolates}

Thirty-two of the 33 AZM-LLR isolates (97.0 \%) contained the wild-type sequence in domain $\mathrm{V}$ of all four $23 \mathrm{~S}$ rRNA alleles, except one isolate $(3.0 \%, 1 / 33)$ that contained mutation C2611T (Escherichia coli numbering; also known as C2599T, N. gonorrhoeae numbering) in a single allele (Table 2 and Additional file 1: Table S1, S2). Thirty-eight of the 44 AZM-MLR isolates $(86.4 \%)$ contained the wild-type sequence in all four $23 \mathrm{~S}$ rRNA alleles, whereas only one isolate $(2.2 \%, 1 / 44$; its $\mathrm{AZM}$ MIC $=2 \mathrm{mg} / \mathrm{L}$ ) contained mutation $\mathrm{C} 2611 \mathrm{~T}$ in a single allele and five isolates $(11.4 \%, 5 / 44$; their AZM MICs $=4,8,8,8$ and $64 \mathrm{mg} / \mathrm{L}$, respectively) contained mutation C2611T in all four alleles. All seven AZM-R isolates that contained the mutation $\mathrm{C} 2611 \mathrm{~T}$ showed reduced susceptibility to ceftriaxone (MIC $\geq 0.125 \mathrm{mg} / \mathrm{L}$ ).

The presence of a single nucleotide deletion (A) in the $13 \mathrm{bp}$ inverted repeat of the $m t r R$ promoter did not different between AZM-LLR $(87.9 \%, 29 / 33)$ and AZMMLR $(95.5 \%, 42 / 44)$ isolates $(P>0.05)$ (Table 2 and Additional file 1: Table S1, S2). A wild type (WT) in the coding region of $m$ trR only displayed in combined AZM-LLR/susceptibility to ceftriaxone $\left(\mathrm{CRO}^{\mathrm{S}}\right)(n=2)$ and combined AZM-MLR/CRO ${ }^{\mathrm{S}}(n=2)$, but not in combined AZM-LLR/CRO ${ }^{\mathrm{D}}$ or combined AZM-MLR/ $\mathrm{CRO}^{\mathrm{D}}$ isolates. The combined mutations (double or triple) such as A39T/F62L, A40D/T86A, T86A/H105Y, A40D/T86A/H105Y or D79N/T86A/H105Y were only present in AZM-MLR isolates (Table 2).

All the AZM-R isolates $(n=77)$ in this study had an Asp-346 insertion and contained F504L, A510V and A516G mutations in PBP2 (data shown in Additional file 1: Table S1-S3). Thirteen mutation patterns in this study

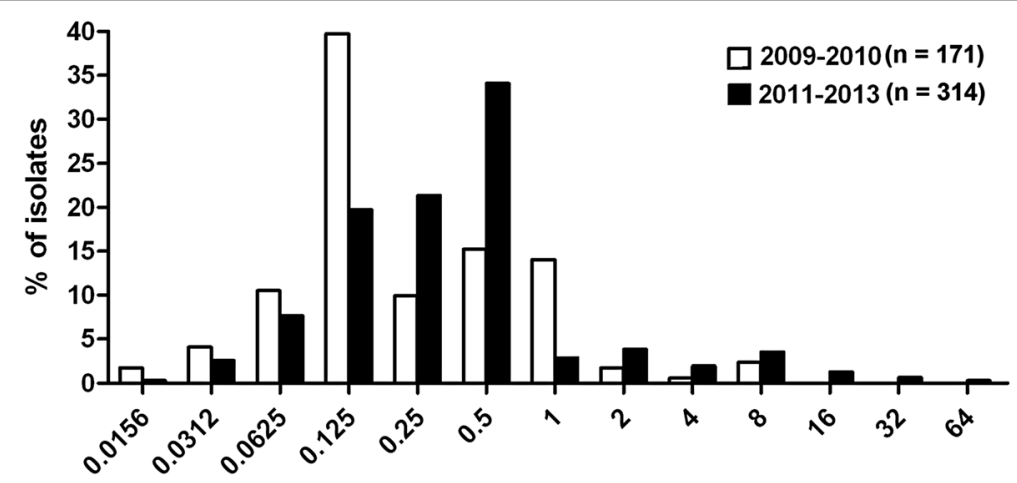

Fig. 1 Distribution of MIC of AZM-R N. gonorrhoeae isolates during 2009 and $2010(n=171)$ versus 2011-2013 $(n=314)$ 
Table 2 Characteristics of N. gonorrhoeae isolates with AZM-R and CROD identified in Guangzhou in 2009-2013

\begin{tabular}{|c|c|c|c|c|c|}
\hline & \multicolumn{2}{|c|}{ AZM-LLR $(n=33)$} & \multicolumn{2}{|c|}{ AZM-MLR $(n=44)$} & \multirow[b]{3}{*}{$P$} \\
\hline & $\overline{\text { Total }^{c}}$ & $\mathrm{CRO}^{\mathrm{D}}$ & $\overline{\text { Total }^{c}}$ & $\mathrm{CRO}^{\mathrm{D}}$ & \\
\hline & $n(\%)$ & $n(\%)$ & $n(\%)$ & $n(\%)$ & \\
\hline Antimicrobial resistance & $33(100.0)$ & $6(18.2)$ & $44(100)$ & $19(43.2)$ & $b<0.05$ \\
\hline \multicolumn{6}{|l|}{$23 \mathrm{~S}$ rRNA } \\
\hline WT & $32(97.0)$ & $5(15.2)$ & $38(86.3)$ & $13(29.5)$ & \multirow[t]{3}{*}{$a, b>0.05$} \\
\hline $\mathrm{C} 2611 \mathrm{~T}^{\mathrm{S}}$ & $1(3.0)$ & $1(3.0)$ & $1(2.3)$ & $1(2.3)$ & \\
\hline $\mathrm{C} 2611 \mathrm{~T}^{\text {All }}$ & $0(0.0)$ & $0(0.0)$ & $5(11.4)$ & $5(11.4)$ & \\
\hline \multicolumn{6}{|l|}{$m t r R$} \\
\hline \multicolumn{6}{|l|}{ Deletion $^{a}$} \\
\hline Yes & $29(87.9)$ & $6(18.2)$ & $42(95.5)$ & $19(43.3)$ & \multirow[t]{2}{*}{$a>0.05, b: N D$} \\
\hline No & $4(12.1)$ & $0(0.0)$ & $2(4.5)$ & $0(0.0)$ & \\
\hline \multicolumn{6}{|l|}{ Mutations } \\
\hline$W T^{b}$ & $2(6.1)$ & $0(0.0)$ & $2(4.5)$ & $0(0.0)$ & \multirow[t]{9}{*}{$a, b>0.05$} \\
\hline A39T & $7(21.2)$ & $4(12.1)$ & $6(13.6)$ & $4(9.1)$ & \\
\hline G45D & $8(24.2)$ & $1(3.0)$ & $5(11.3)$ & $2(4.5)$ & \\
\hline $\mathrm{H} 105 \mathrm{Y}$ & $16(48.5)$ & $1(3.0)$ & $26(59.1)$ & $11(25.0)$ & \\
\hline A39T/ F62L & $0(0.0)$ & $0(0.0)$ & $1(2.3)$ & $0(0.0)$ & \\
\hline $\mathrm{A} 40 \mathrm{D} / \mathrm{T} 86 \mathrm{~A}$ & $0(0.0)$ & $0(0.0)$ & $1(2.3)$ & $0(0.0)$ & \\
\hline $\mathrm{A} 40 \mathrm{D} / \mathrm{T} 86 \mathrm{~A} / \mathrm{H} 105 \mathrm{Y}$ & $0(0.0)$ & $0(0.0)$ & $1(2.3)$ & $1(2.3)$ & \\
\hline D79N/T86A/H105Y & $0(0.0)$ & $0(0.0)$ & $1(2.3)$ & $1(2.3)$ & \\
\hline T86A/ H105Y & $0(0.0)$ & $0(0.0)$ & $1(2.3)$ & $0(0.0)$ & \\
\hline \multicolumn{6}{|l|}{ Mutation patterns in PBP2 } \\
\hline II or XIV & $3(9.1)$ & $0(0.0)$ & $4(9.1)$ & $0(0.0)$ & \multirow[t]{13}{*}{$a, b>0.05$} \\
\hline V & $6(18.2)$ & $1(3.0)$ & $8(18.2)$ & $3(6.8)$ & \\
\hline $\mathrm{VII}$ & $0(0.0)$ & $0(0.0)$ & $1(2.3)$ & $0(0.0)$ & \\
\hline VIII & $0(0.0)$ & $0(0.0)$ & $1(2.3)$ & $1(2.3)$ & \\
\hline$X \|$ & $2(6.1)$ & $0(0.0)$ & $2(4.6)$ & $1(2.3)$ & \\
\hline$X I I I$ & $2(6.1)$ & $0(0.0)$ & $0(0.0)$ & $0(0.0)$ & \\
\hline$X V I I$ & $2(6.1)$ & $1(3.0)$ & $0(0.0)$ & $0(0.0)$ & \\
\hline $\mathrm{XVIII}$ & $5(15.2)$ & $2(6.1)$ & $13(29.4)$ & $10(22.6)$ & \\
\hline$X X I$ & $7(21.2)$ & $1(3.0)$ & $8(18.2)$ & $3(6.8)$ & \\
\hline$X X V I I$ & $4(12.2)$ & $0(0.0)$ & $6(13.6)$ & $1(2.3)$ & \\
\hline New $1^{d}$ & $1(3.0)$ & $0(0.0)$ & $0(0.0)$ & $0(0.0)$ & \\
\hline New $2^{d}$ & $1(3.0)$ & $1(3.0)$ & $0(0.0)$ & $0(0.0)$ & \\
\hline New $3^{d}$ & $0(0.0)$ & $0(0.0)$ & $1(2.3)$ & $0(0.0)$ & \\
\hline \multicolumn{6}{|c|}{ 23rRNA/mtrR/penA mutation patterns } \\
\hline WT/A-;H105Y/XVIII & $2(6.1)$ & $0(0.0)$ & $4(9.1)$ & $3(6.8)$ & \multirow[t]{5}{*}{$a, b>0.05$} \\
\hline WT/A-;H105YN & $6(18.2)$ & $1(3.0)$ & $6(13.6)$ & $3(6.8)$ & \\
\hline WT/A-;H105Y/XXI & $2(6.1)$ & $0(0.0)$ & $6(13.6)$ & $2(4.5)$ & \\
\hline WT/A-;G45D/XXI & $4(12.1)$ & $3(9.1)$ & $0(0.0)$ & $0(0.0)$ & \\
\hline WT/A-;A39T/XVIII & $3(9.1)$ & $3(9.1)$ & $5(11.4)$ & $3(6.8)$ & \\
\hline
\end{tabular}


Table 2 Characteristics of N. gonorrhoeae isolates with AZM-R and CROD identified in Guangzhou in 2009-2013 (Continued)

\begin{tabular}{|c|c|c|c|c|}
\hline $\mathrm{C} 2611 \mathrm{~T}^{\mathrm{All}} / \mathrm{A}-\mathrm{-H} 105 \mathrm{Y} / \mathrm{XVIII}$ & $0(0.0)$ & $0(0.0)$ & $2(4.5)$ & $2(4.5)$ \\
\hline $\mathrm{C} 2611 \mathrm{~T}^{\mathrm{All}} / \mathrm{A}-; \mathrm{G} 45 \mathrm{D} / \mathrm{XXI}$ & $0(0.0)$ & $0(0.0)$ & $2(4.5)$ & $2(4.5)$ \\
\hline Other patterns & $16(48.5)$ & $2(6.1)$ & $17(38.6)$ & $2(4.5)$ \\
\hline
\end{tabular}

AZM azithromycin, $C R O^{D}$ decreased susceptibility to ceftriaxone, $C R O^{S}$ susceptible to ceftriaxone, $C 2611 T^{S}$ mutation $C 2611 \mathrm{~T}$ in a single allele, $C 2611 T^{A l l} \mathrm{C} 2611 \mathrm{~T}$ mutations in all four alleles, ${ }^{a}$ Adenine (A) deletion in the 13-bp inverted repeat (5'-AAAAAGACTTITT-3') within the -35 to - 10 positions of the $m$ trR promoter. ${ }^{\text {b }}$ Using $m$ trR from N. gonorrhoeae FA1090 as a template. ' Including CRO ${ }^{\text {S }}$ and CRO ${ }^{\text {; }}{ }^{d}$ New polymorphism patterns of PBP2: New 1 (A501V, F504L, A510V, A516G, H541N, P552V, I556V, I566V), New 2 (F504L, A510V, A516G, Y554H), New 3 (A501V, F504L, A510V, A516G, H541N, I566V). a: the AZM-LLR isolates were compared to the AZM-MLR isolates; b: the combined $A Z M-L L R / C R O^{D}$ isolates were compared to the combined $A Z M-M L R / C R O^{D}$ isolates; $N D$ not determined (because of a small number of isolates)

included three new mutation patterns (New 1: A501V, F504L A501V, A516G, H541N, P552V, I556V, I566V; New 2: F504L, A501V, A516G, Y554H; New 3: A501V, F504L, A510V, A516G, H541N, I566V) were shown in Table 2. The patterns V (18.2\%), XVIII (15.2\%) and XXI (21.2 \%) were the most prevalent in AZM-LLR isolates, similar to the most prevalent patterns $(\mathrm{V}, 18.2 \%$; XVIII, 29.4 \%; XXI, 18.2 \%) in AZM-MLR isolates. No different mutation patterns were found between AZM-LLR isolates and AZM-MLR isolates or between combined AZM-LLR/CRO ${ }^{\mathrm{D}}$ isolates and combined AZM-MLR/CRO ${ }^{\mathrm{D}}$ isolates (both $P>0.05$ ).

In addition, combined $23 \mathrm{~S} \mathrm{rRNA} / \mathrm{mtrR} /$ penA mutation patterns had no significant association with AZM-MLR isolates or combined AZM-MLR/CRO ${ }^{\mathrm{D}}$ isolates (Table 2).

\section{NG-MAST and phylogenetic analysis based on porB and tbpB DNA sequences}

A total of 67 STs (29 STs in AZM-LLR isolates and 38 STs in AZM-MLR isolates) were identified by NGMAST among the 77 AZM-R isolates, of which 30 STs (15 STs in AZM-LLR isolates and 15 STs in AZM-MLR isolates) were first identified by the current study (Table 3 and Additional file 1: Table S1, S2 and S3). The most prevalent STs were ST1766 $(n=3), \operatorname{ST} 1866(n=2)$, and ST421 $(n=2)$ in AZM-LLR isolates and ST1766 $(n=$ $3)$ ST6987 $(n=2), \operatorname{ST} 1055(n=2), \operatorname{ST} 304(n=2)$, and a new ST consisting of porB allele 822 and tbpB allele 156 $(n=2)$ in AZM-MLR isolates. Of the remaining STs, most were represented by a single isolate. The phylogenetic tree, built from DNA sequences of por (490 bp) and $t b p B$ (390 bp), displayed the significant diversity of strains with 29 different STs in AZM-LLR isolates (Additional file 2: Figure S1), as well as 38 different STs in AZM-MLR isolates (Additional file 3: Figure S2).

\section{Discussion}

The present study combines antimicrobial susceptibility determinations with molecular-based analysis of AZM-R in N. gonorrhoeae isolated from 2009 to 2013 in Guangzhou, China. As observed in other countries worldwide $[9,13,18]$, an upward shift in MIC by year and a high percentage of AZM-R were identified in this study, which was also in line with a previous report in two Chinese cities, Nanjing and Chongqing, between 2008 and 2009 [5]. The WHO recommends discontinuation of the empirical use of an antibiotic once $5 \%$ of locally acquired gonococcal isolates are resistant [19]. Accordingly, AZM has not been recommended as a monotherapy for gonococcal urethritis or cervicitis in China, as well as in many other countries worldwide. To improve treatment efficacy and to delay the further selection of cephalosporin-resistant $N$. gonorrhoeae, most current guidelines now recommend ESCs and AZM in combination as the first-line treatment for gonorrhea [2-4, 9]. However, more recently, AZM-R N. gonorrhoeae has been rarely and sporadically reported to also have reduced susceptibility to ESCs $[9,13]$, threatening the future efficacy of current therapeutic recommendations. Similarly, the present study showed that the prevalence of combined AZM-MLR/CRO ${ }^{\mathrm{D}}$ isolates significantly increased as compared with that in all identified N. gonorrhoeae isolates.

Previous studies investigating AZM-R and $C R \mathrm{O}^{\mathrm{D}}$ have focused on the mutations of three genes: 23S rRNA, $m t r R$ and penA [9-12]. Mutations at various positions within the central loop of domain $\mathrm{V}$ of the $23 \mathrm{~S}$ rRNA gene are thought to lead to resistance by reducing the ability of the 23S rRNA protein to bind the antibiotics such as AZM [10]. Previous reports have shown that the mutation C2611T in any of the four alleles of the 23S rRNA can result in a lower level of resistance to AZM [10]. In addition, the mutation A2059G (Escherichia coli numbering) in at least three of the four alleles can confer AZM-HLR, and AZM-sensitive isolates containing this single allele mutation can quickly develop high-level resistance in the presence of erythromycin [10]. In the current study, only seven AZM-R isolates contained mutation $\mathrm{C} 2611 \mathrm{~T}$, whereas the remaining large number of AZM-R isolates contained four wild-type alleles. These findings contrast, however, with other reports from Canada [9], the United States [11, 20] and the United Kingdom [10], which most of the AZM-MLR isolates contained this mutation was more than $80 \%$. Notably, all the above-mentioned seven AZM-R isolates that contained the mutation $\mathrm{C} 2611 \mathrm{~T}$ showed reduced susceptibility to ceftriaxone. The mutation A2059G, which is linked to AZM-HLR, was not detected in the 
Table 3 NG-MAST STs and N. gonorrhoeae isolated with AZM-R and $C^{D}{ }^{D}$ identified in Guangzhou in 2009-2013

\begin{tabular}{|c|c|c|c|c|}
\hline \multirow[b]{3}{*}{ NG-MAST ST } & \multicolumn{2}{|c|}{ AZM-LLR $(n=33)$} & \multicolumn{2}{|c|}{ AZM-MLR $(n=44)$} \\
\hline & Total $^{b}$ & $C R O^{D}$ & Total $^{b}$ & $\mathrm{CRO}^{\mathrm{D}}$ \\
\hline & $n(\%)$ & $n(\%)$ & $n(\%)$ & $n(\%)$ \\
\hline 270 & $0(0.0)$ & $0(0.0)$ & $1(2.3)$ & $1(2.3)$ \\
\hline 304 & $0(0.0)$ & $0(0.0)$ & $2(4.5)$ & $0(0.0)$ \\
\hline 421 & $2(6.1)$ & $0(0.0)$ & $0(0.0)$ & $0(0.0)$ \\
\hline 1053 & $1(3.0)$ & $0(0.0)$ & $1(2.3)$ & $1(2.3)$ \\
\hline 1055 & $0(0.0)$ & $0(0.0)$ & $2(4.5)$ & $0(0.0)$ \\
\hline 1056 & $0(0.0)$ & $0(0.0)$ & $1(2.3)$ & $0(0.0)$ \\
\hline 1412 & $1(3.0)$ & $0(0.0)$ & $0(0.0)$ & $0(0.0)$ \\
\hline 1731 & $1(3.0)$ & $0(0.0)$ & $0(0.0)$ & $0(0.0)$ \\
\hline 1766 & $3(9.1)$ & $0(0.0)$ & $3(6.8)$ & $0(0.0)$ \\
\hline 1866 & $2(6.1)$ & $0(0.0)$ & $1(2.3)$ & $0(0.0)$ \\
\hline 1972 & $1(3.0)$ & $1(3.0)$ & $0(0.0)$ & $0(0.0)$ \\
\hline 2103 & $1(3.0)$ & $0(0.0)$ & $0(0.0)$ & $0(0.0)$ \\
\hline 2384 & $0(0.0)$ & $0(0.0)$ & $1(2.3)$ & $1(2.3)$ \\
\hline 3079 & $1(3.0)$ & $0(0.0)$ & $0(0.0)$ & $0(0.0)$ \\
\hline 3252 & $0(0.0)$ & $0(0.0)$ & $1(2.3)$ & $1(2.3)$ \\
\hline 3356 & $0(0.0)$ & $0(0.0)$ & $1(2.3)$ & $1(2.3)$ \\
\hline 3460 & $1(3.0)$ & $0(0.0)$ & $0(0.0)$ & $0(0.0)$ \\
\hline 4313 & $0(0.0)$ & $0(0.0)$ & $1(2.3)$ & $1(2.3)$ \\
\hline 4539 & $1(3.0)$ & $0(0.0)$ & $0(0.0)$ & $0(0.0)$ \\
\hline 5061 & $0(0.0)$ & $0(0.0)$ & $1(2.3)$ & $1(2.3)$ \\
\hline 5062 & $0(0.0)$ & $0(0.0)$ & $1(2.3)$ & $0(0.0)$ \\
\hline 5179 & $1(3.0)$ & $1(3.0)$ & $0(0.0)$ & $0(0.0)$ \\
\hline 5990 & $0(0.0)$ & $0(0.0)$ & $1(2.3)$ & $0(0.0)$ \\
\hline 6987 & $0(0.0)$ & $0(0.0)$ & $2(4.5)$ & $1(2.3)$ \\
\hline 7101 & $0(0.0)$ & $0(0.0)$ & $1(2.3)$ & $1(2.3)$ \\
\hline 8776 & $1(3.0)$ & $0(0.0)$ & $0(0.0)$ & $0(0.0)$ \\
\hline 9176 & $0(0.0)$ & $0(0.0)$ & $1(2.3)$ & $1(2.3)$ \\
\hline 9944 & $1(3.0)$ & $0(0.0)$ & $0(0.0)$ & $0(0.0)$ \\
\hline 10199 & $0(0.0)$ & $0(0.0)$ & $1(2.3)$ & $1(2.3)$ \\
\hline 10205 & $0(0.0)$ & $0(0.0)$ & $1(2.3)$ & $1(2.3)$ \\
\hline 10337 & $0(0.0)$ & $0(0.0)$ & $1(2.3)$ & $0(0.0)$ \\
\hline 10352 & $0(0.0)$ & $0(0.0)$ & $1(2.3)$ & $0(0.0)$ \\
\hline 10359 & $0(0.0)$ & $0(0.0)$ & $1(2.3)$ & $0(0.0)$ \\
\hline 11181 & $0(0.0)$ & $0(0.0)$ & $1(2.3)$ & $0(0.0)$ \\
\hline $\mathrm{New}^{\mathrm{a}}$ & $15(45.5)$ & $4(12.1)$ & 16 (36.4) & 7 (15.9) \\
\hline
\end{tabular}

$A Z M$ azithromycin, $C R O^{D}$ decreased susceptibility to ceftriaxone, $C R O^{5}$ susceptible to ceftriaxone, ${ }^{a}$ Not found in the NG-MAST database (http://www.ng-mast.net); ${ }^{\mathrm{b}}$ Including $\mathrm{CRO}^{\mathrm{S}}$ and $\mathrm{CRO}^{\mathrm{D}}$

present study. In the $m t r R$ gene, specific mutations in the promoter or coding region can lead to decrease the MtrCDE efflux pump repression and subsequently increase export of the antimicrobials [20]. Loss of the
MtrCDE efflux pump can significantly increase susceptibility to AZM, ESCs, penicillin, ciprofloxacin, and solithromyc in $N$. gonorrhoeae isolates [21]. In this study, a single nucleotide deletion in the mtrR promoter showed over $85 \%$ in AZMR-R isolates, but not significantly difference between combined AZMR-R/ $\mathrm{CRO}^{\mathrm{S}}$ isolates and combined AZMR-R/CRO ${ }^{\mathrm{D}}$ isolates, in line with a previous report that such a deletion may not implicate in $\mathrm{CRO}^{\mathrm{D}}$ [12]. H105Y, the most prevalent single mutation, was not significantly difference in AZM-LLR isolates as compared to AZM-MLR isolates. Interestingly, the combined mutation in $m t r R$ was only observed in AZM-MLR isolates, but not in in AZM-LLR isolates. Mutation in penA has been associated with decreased susceptibility or resistance to a number of beta-lactam agents, especially to ESCs [22]. The mutations G545S, I312M, and V316T in mosaic alleles of PBP2 were early suggested as important for the increased ESCs resistance [23]. However, a recent study revealed that these three mutations had only a little to effect on resistance, and their capacity to increase resistance to ESCs was dependent on the presence of other mutations in the mosaic alleles [24]. Mutation patterns XIII, XVII and XVIII have been reported to only present in $\mathrm{CRO}^{\mathrm{D}}$ isolates in China [12] and Australia [22]. These three patterns contained G542S, P551S and/or P551L mutations, which have been associated with increased MIC of CRO [25]. However, their effects on the ceftriaxone MIC have not yet been proven by site-directed penA mutations in isogenic strain backgrounds [26] and the present study showed that these three patterns were present in both $\mathrm{CRO}^{\mathrm{S}}$ and $\mathrm{CRO}^{\mathrm{D}}$ isolates (data not shown). The most prevalent mutation pattern in AZMR-R isolates from Canada was XXXIV, which was showed to AZM$\mathrm{R}$ together with reduced susceptibility to ESCs [9]. By comparison, this study revealed the most prevalent mutation patterns (V, XVIII and XXI) were the same in AZMR-LLR, AZMR-MLR, and combined AZMR-R/ $\mathrm{CRO}^{\mathrm{D}}$ isolates, respectively. Overall, the results of our study showed that the mutations of these three genes had the considerable diversity, and that either one of these three genes alone or combined mutation patterns of these three genes cannot account for AZM-MLR or combined AZM-R/CRO ${ }^{\mathrm{D}}$ in our $N$. gonorrhoeae isolates.

NG-MAST analysis and the resulting development of a phylogenetic tree in this study also indicated a high degree of genetic diversity of $N$. gonorrhoeae in Guangzhou from 2009 to 2013. This observation may be due to the highly limited epidemiologic information of this study because our data do not represent the whole country, or the flow of large numbers of temporary population in Guangzhou, which lead to great 
opportunity for transport of new STs [27]. Also, AZM is extensively used in China [5] and has long elimination half-life. Thus we postulate that the diversity of STs developed, in part, in response to antibiotic pressure. Some STs that were observed in the current study (ST3356, ST1866, ST1766, and ST4313) had been noted in a previous report of emerging AZM-R N. gonorrhoeae in Nanjing and Chongqing, China [5], but these did not include STs (ST470, ST649, ST3158, ST1704, ST359, and ST696) that were reported previously in other countries and have been shown to account for most of the resistance or high-level resistance to AZM [9, 18, 28-30].

\section{Conclusions}

AZM-R N. gonorrhoeae isolates are increasing and, in combination with $\mathrm{CRO}^{\mathrm{D}}$, have now been detected in Guangzhou. The combined AZM-R/CRO ${ }^{\mathrm{D}}$ in $N$. gonorrhoeae is a relatively new phenomenon, and the mechanism for the emergence of these $\mathrm{AZM}-\mathrm{R} / \mathrm{CRO}^{\mathrm{D}}$ isolates remains unknown and needs further studied. Therefore, antimicrobial susceptibility/resistance and the molecular epidemiology of AZM-R and/or $\mathrm{CRO}^{\mathrm{D}}$ deserve continuous surveillance, which will be critical in providing valuable information for current and future therapeutic options in China.

\section{Availability of data and materials}

The sequencing datasets of porB, tbpB, 23S rRNA, mtrR and penA genes supporting the conclusions of this article are available in the Dryad Digital Repository: http:// dx.doi.org/10.5061/dryad.cp3d8.

\section{Additional files}

Additional file 1: Table S1. Characteristics of AZM-LLR N. gonorrhoeae isolates identified in Guangzhou in 2009-2013 ( $n=33)$. Table S2. Characteristics of AZM-MLR N. gonorrhoeae isolates identified in Guangzhou in 2009-2013 $(n=44)$. Table S3. The classification of mutation patterns of the PBP2 proteins based on amino acids 500 to 570. (DOCX $46.2 \mathrm{~kb}$ )

Additional file 2: Figure S1. A neighbor-joining phylogenetic tree was constructed using the concatenated sequences of por (490 bp) and tbpB (390 bp) alleles, which were identified by the NG-MAST method, from 33 AZM-LLR N. gonorrhoeae isolates in Guangzhou, China in 2009-2013. (DOCX $1475 \mathrm{~kb}$ )

Additional file 3: Figure S2. A neighbor-joining phylogenetic tree was constructed using the concatenated sequences of por (490 bp) and tbpB (390 bp) alleles, which were identified by the NG-MAST method, from 44 AZM-MLR N. gonorrhoeae isolates in Guangzhou, China in 2009-2013. (DOCX $1372 \mathrm{~kb})$

\section{Abbreviations}

AZM: azithromycin; AZM-HLR: azithromycin high-level resistant; AZMLLR: azithromycin low-level resistant; AZM-MLR: azithromycin middle-level resistant; AZM-R: azithromycin-resistant; $C R O D$ : decreased susceptibility to ceftriaxone; $\mathrm{CRO}^{\text {S}}$ : susceptibility to ceftriaxone; ESCs: extended-spectrum cephalosporins; MIC: minimum inhibitory concentration; NG-MAST: N. gonorrhoeae multi-antigen sequence typing; PBP2: penicillin-binding protein 2; STI: sexually transmitted infection..

\section{Competing interests}

The authors declare that they have no competing interests.

\section{Authors' contributions}

$J Y L$ and WLC contributed to the design of the analysis and wrote the draft of the manuscript. YRD, XDY and XXC provided $N$. gonorrhoeae isolates and clinical information. XDL, CB, YHL and PL identified $N$. gonorrhoeae isolates and tested antimicrobial susceptibility. XBZ is the corresponding author, supervised the research and edited the manuscript. All authors read and approved the final manuscript.

\section{Acknowledgments}

We are grateful for the collaboration of the heads and staff at STI clinics in Guangzhou, China.

Received: 22 October 2015 Accepted: 14 March 2016 Published online: 14 April 2016

\section{References}

1. Kovari $H$, de Melo Oliveira MD, Hauser P, Lauchli S, Meyer J, Weber R, Zbinden R. Decreased susceptibility of Neisseria gonorrhoeae isolates from Switzerland to Cefixime and Ceftriaxone: antimicrobial susceptibility data from 1990 and 2000 to 2012. BMC Infect Dis. 2013;13:603.

2. Centers for Disease C, Prevention. Update to CDC's Sexually transmitted diseases treatment guidelines, 2010: oral cephalosporins no longer a recommended treatment for gonococcal infections. MMWR Morb Mortal Wkly Rep. 2012;61(31):590-4.

3. Bignell C, Unemo M, European STIGEB. 2012 European guideline on the diagnosis and treatment of gonorrhoea in adults. Int J STD AIDS. 2013;24(2):85-92.

4. Chen SC, Yin YP, Dai XQ, Unemo M, Chen XS. Antimicrobial resistance, genetic resistance determinants for ceftriaxone and molecular epidemiology of Neisseria gonorrhoeae isolates in Nanjing, China. J Antimicrob Chemother. 2014:69(11):2959-65.

5. Yuan LF, Yin YP, Dai XQ, Pearline RV, Xiang Z, Unemo M, Chen XS. Resistance to azithromycin of Neisseria gonorrhoeae isolates from 2 cities in China. Sex Transm Dis. 2011;38(8):764-8.

6. Yu LH, Qu Y. Analysis of antimicrobial susceptibilities of 201 Neisseria gonorrhoeae strains [in Chinese]. Zhejiang Pract Med. 2004;9:60-1.

7. Cao WL, LI XD, Bi C, Liang YH, Song WZ, Lin LY, Wu DB, Zhang L. Analysis of the drug-resistance of Neisseria gonorrhoeae to 6 types of antibiotics in certain area. Int J Lab Med. 2011;32(19):2205-6.

8. Morita-Ishihara T, Unemo M, Furubayashi K, Kawahata T, Shimuta K, Nakayama S, Ohnishi M. Treatment failure with $2 \mathrm{~g}$ of azithromycin (extended-release formulation) in gonorrhoea in Japan caused by the international multidrugresistant ST1407 strain of Neisseria gonorrhoeae. J Antimicrob Chemother. 2014;69(8):2086-90.

9. Allen VG, Seah C, Martin I, Melano RG. Azithromycin resistance is coevolving with reduced susceptibility to cephalosporins in Neisseria gonorrhoeae in Ontario, Canada. Antimicrob Agents Chemother. 2014;58(5):2528-34.

10. Chisholm SA, Dave J, Ison CA. High-level azithromycin resistance occurs in Neisseria gonorrhoeae as a result of a single point mutation in the 235 rRNA genes. Antimicrob Agents Chemother. 2010;54(9):3812-6.

11. Wu A, Buono S, Katz KA, Pandori MW. Clinical Neisseria gonorrhoeae isolates in the United States with resistance to azithromycin possess mutations in all $23 \mathrm{~S}$ rRNA alleles and the mtrR coding region. Microb Drug Resist. 2011;17(3):425-7.

12. Liao M, Gu WM, Yang Y, Dillon JA. Analysis of mutations in multiple loci of Neisseria gonorrhoeae isolates reveals effects of PIB, PBP2 and MtrR on reduced susceptibility to ceftriaxone. J Antimicrob Chemother. 2011:66(5):1016-23.

13. Katz AR, Komeya AY, Soge OO, Kiaha MI, Lee MV, Wasserman GM, Maningas EV, Whelen AC, Kirkcaldy RD, Shapiro SJ et al. Neisseria gonorrhoeae with highlevel resistance to azithromycin: case report of the first isolate identified in the United States. Clin Infect Dis. 2012;54(6):841-3.

14. Cao WL, Liang JY, Li XD, Bi C, Yang RD, Liang YH, Li P, Zhong DQ, Ye XD, Zhang XB. Trends in antimicrobial resistance in Neisseria gonorrhoeae isolated from Guangzhou, China, 2000 to 2005 and 2008 to 2013. Sex Transm Dis. 2015;42(1):27-9.

15. World Health Organization. Global action plan to control the spread and impact of antimicrobial resistance in Neisseria gonorrhoeae. 2012. Available at: http://www.who.int/reproductivehealth/publications/rtis/9789241503501/ en/. (19 October 2015, date last accessed). 
16. Ng LK, Martin I, Liu G, Bryden L. Mutation in $23 \mathrm{~S}$ rRNA associated with macrolide resistance in Neisseria gonorrhoeae. Antimicrob Agents Chemother. 2002;46(9):3020-5.

17. Martin IM, Ison CA, Aanensen DM, Fenton KA, Spratt BG. Rapid sequence-based identification of gonococcal transmission clusters in a large metropolitan area. J Infect Dis. 2004;189(8):1497-505.

18. Starnino S, Stefanelli P, Neisseria gonorrhoeae Italian Study G. Azithromycinresistant Neisseria gonorrhoeae strains recently isolated in Italy. J Antimicrob Chemother. 2009;63(6):1200-4.

19. Kaiser NC, Liang L, Melrose RJ, Wilkins SS, Sultzer DL, Mendez MF. Differences in anxiety among patients with early- versus late-onset Alzheimer's disease. J Neuropsychiatry Clin Neurosci. 2014;26(1):73-80.

20. Centers for Disease C, Prevention. Neisseria gonorrhoeae with reduced susceptibility to azithromycin-San Diego County, California, 2009. MMWR Morb Mortal Wkly Rep. 2011:60(18):579-81.

21. Golparian D, Shafer WM, Ohnishi M, Unemo M. Importance of multidrug efflux pumps in the antimicrobial resistance property of clinical multidrugresistant isolates of Neisseria gonorrhoeae. Antimicrob Agents Chemother. 2014;58(6):3556-9.

22. Whiley DM, Limnios EA, Ray S, Sloots TP, Tapsall JW. Diversity of penA alterations and subtypes in Neisseria gonorrhoeae strains from Sydney, Australia, that are less susceptible to ceftriaxone. Antimicrob Agents Chemother. 2007;51(9):3111-6.

23. Takahata S, Senju N, Osaki Y, Yoshida T, Ida T. Amino acid substitutions in mosaic penicillin-binding protein 2 associated with reduced susceptibility to cefixime in clinical isolates of Neisseria gonorrhoeae. Antimicrob Agents Chemother. 2006;50(11):3638-45.

24. Tomberg J, Unemo M, Davies C, Nicholas RA. Molecular and structural analysis of mosaic variants of penicillin-binding protein 2 conferring decreased susceptibility to expanded-spectrum cephalosporins in Neisseria gonorrhoeae: role of epistatic mutations. Biochemistry. 2010;49(37):8062-70.

25. Whiley DM, Goire N, Lambert SB, Ray S, Limnios EA, Nissen MD, Sloots TP, Tapsall JW. Reduced susceptibility to ceftriaxone in Neisseria gonorrhoeae is associated with mutations G542S, P551S and P551L in the gonococcal penicillin-binding protein 2. J Antimicrob Chemother. 2010;65(8):1615-8.

26. Unemo M, Shafer WM. Antimicrobial resistance in Neisseria gonorrhoeae in the 21st century: past, evolution, and future. Clin Microbiol Rev. 2014;27(3):587-613.

27. Wenling C, Xibao Z, Shi F, Minchang W, Ping L, Debiao W, Jinlan Y. Analysis of the antibiotic sensitivity of Neisseria gonorrhoeae in Guangzhou, Peoples Republic of China. Sex Transm Dis. 2000;27(8):480-2.

28. Palmer HM, Young H, Winter A, Dave J. Emergence and spread of azithromycin-resistant Neisseria gonorrhoeae in Scotland. J Antimicrob Chemother. 2008;62(3):490-4.

29. Chisholm SA, Neal TJ, Alawattegama AB, Birley HD, Howe RA, Ison CA. Emergence of high-level azithromycin resistance in Neisseria gonorrhoeae in England and Wales. J Antimicrob Chemother. 2009;64(2):353-8.

30. Galarza PG, Alcala B, Salcedo C, Canigia LF, Buscemi L, Pagano I, Oviedo C, Vazquez JA. Emergence of high level azithromycin-resistant Neisseria gonorrhoeae strain isolated in Argentina. Sex Transm Dis. 2009;36(12):787-8.

\section{Submit your next manuscript to BioMed Central and we will help you at every step:}

- We accept pre-submission inquiries

- Our selector tool helps you to find the most relevant journal

- We provide round the clock customer support

- Convenient online submission

- Thorough peer review

- Inclusion in PubMed and all major indexing services

- Maximum visibility for your research

Submit your manuscript at www.biomedcentral.com/submit

) Biomed Central 